\title{
CONSTRUCTION OF ATTRACTORS AND FILTRATIONS
}

\author{
GEORGE OSIPENKO \\ Laboratory of Nonlinear Analysis and Mathematical Modelling \\ St. Petersburg State Technical University, St. Petersburg 195251, Russia \\ E-mail: math@math.hop.stu.neva.ru
}

\begin{abstract}
This paper is a study of the global structure of the attractors of a dynamical system. The dynamical system is associated with an oriented graph called a Symbolic Image of the system. The symbolic image can be considered as a finite discrete approximation of the dynamical system flow. Investigation of the symbolic image provides an opportunity to localize the attractors of the system and to estimate their domains of attraction. A special sequence of symbolic images is considered in order to obtain precise knowledge about the global structure of the attractors and to get filtrations of the system.
\end{abstract}

Introduction. Our purpose is to study the structure of attractors without any preliminary information about the system. The investigation is based on the methods of symbolic dynamics and all needed estimations can be obtained by traditional numerical methods. The common scheme of the investigation is the following. By using a covering of phase space by cells the dynamical system is associated with an oriented graph called the Symbolic Image of the system. Valuable information about the global structure of the system may come from analysis of this symbolic image. By investigating the symbolic image, one can obtain neighborhoods of the attractors and estimate their domains of attraction. This allows us to construct a filtration of the dynamical system. By applying a subdivision of the covering, a fine sequence of filtrations is constructed. It must be emphasized that our investigation was stimulated by the basic ideas of Charles Conley [5] of the chain recurrent set, the Morse decomposition and the Lyapunov functions.

We will consider a discrete dynamical system governed by a homeomorphism $X$ defined on a compact $C^{\infty}$ manifold $M$. To describe the continuous version, consider a shift operator along trajectories of the system of differential equations defined as follows. Let $x^{\prime}=f(t, x)$ be a system of ordinary differential equations, where $x \in M, f(t, x)$ is a $C^{1}$

1991 Mathematics Subject Classification: Primary 58F12; Secondary 54H20, 34C35.

Supported by the Russian Foundation for Basic Research under Grant 97-07-90088 and in part by Grant No NWJOOO from the International Science Foundation.

The paper is in final form and no version of it will be published elsewhere. 
vector field periodic in $t$ with period $\omega$. Denote its solution by $\Phi\left(t, t_{0}, x_{0}\right), \Phi\left(t_{0}, t_{0}, x_{0}\right)=$ $x_{0}$. For investigation of the global evolution of the system, it is usually sufficient to examine the Poincaré map $X(x)=\Phi(\omega, 0, x)$ which is the $\omega$-shift operator along the trajectories of the system. If the system of differential equations is autonomous (i.e., the vector field $f$ does not depend on $t$ ), we fix an arbitrary $\omega \neq 0$ and consider a shift operator of the form $X(x)=\Phi(\omega, x)$, where $\Phi(t, x)$ is the solution of the autonomous system, $\Phi(0, x)=x$.

Attractors and $\varepsilon$-trajectories. Consider a discrete dynamical system generated by a homeomorphism $X: M \rightarrow M$ of a compact manifold $M$. Let us denote by $\rho(x, y)$ a distance on $M$. A distance between a point $x$ and a set $A$ is $\rho(x, A)=\inf (\rho(x, y): y \in A)$. Denote by $V(\varepsilon, A)=\{x: \rho(x, A)<\varepsilon\}, \varepsilon>0$ the $\varepsilon$-neighborhood of $A$. Let the trajectory through a point $x$ be $T(x)=\left\{X^{n}(x), n \in \mathbf{Z}\right\}$, the positive semi-trajectory be $T^{+}(x)=$ $\left\{X^{n}(x), n \in \mathbf{Z}^{+}\right\}$, and the negative semi-trajectory be $T^{-}(x)=\left\{X^{n}(x), n \in \mathbf{Z}^{-}\right\}$, where $\mathbf{Z}, \mathbf{Z}^{+}$and $\mathbf{Z}^{-}$are the sets of integers, positive integers and negative integers respectively. A point $y$ belongs to the $\omega$-limit set of $x, \omega(x)$, when there exists a sequence of integers $n_{k} \rightarrow \infty$ such that $X^{n_{k}}(x) \rightarrow y$, i.e.,

$$
\omega(x)=\bigcap_{n>0} \operatorname{cl} X^{n}\left(T^{+}(x)\right)
$$

where $c l A$ means the closure of the set $A$. Analogously, the $\alpha$-limit set of $x, \alpha(x)$, is the set of the limit points of the negative semi-trajectory

$$
\alpha(x)=\bigcap_{n<0} \operatorname{cl} X^{n}\left(T^{-}(x)\right)
$$

Recall that a set $\Lambda$ is invariant if $x \in \Lambda$ implies $T(x) \subset \Lambda$.

Definition 1. An invariant set $\Lambda$ is called Lyapunov stable if for every $\varepsilon>0$ there exists $\delta>0$ such that if $x \in V(\delta, \Lambda)$ then the positive semi-trajectory $T^{+}(x) \subset V(\varepsilon, \Lambda)$.

A stable invariant set $\Lambda$ is said to be asymptotically Lyapunov stable if there is a neighborhood $V$ of $\Lambda$ such that for each $x \in V$

$$
\lim _{n \rightarrow \infty} \rho\left(X^{n}(x), \Lambda\right)=0 .
$$

A closed asymptotically stable set $\Lambda$ is called an attractor.

The set

$$
W^{s}(\Lambda)=\left\{x: \lim _{n \rightarrow \infty} \rho\left(X^{n}(x), \Lambda\right)=0\right\}
$$

is called the domain of attraction of $\Lambda$.

Proposition $1[2,4]$. A closed invariant set $\Lambda$ is an attractor if and only if there is a neighborhood $V$ of $\Lambda$ such that

$$
\Lambda=\bigcap_{n>0} \operatorname{cl} X^{n}(V)
$$

From the definition it follows that $W^{s}(\Lambda)=\{x: \omega(x) \subset \Lambda\}$. The domain of attraction is an invariant set and is a neighborhood of $\Lambda[2,4]$. It is clear that an invariant set for the homeomorphism $X$ is invariant for the inverse mapping $X^{-1}$ as well. 
Definition 2. An invariant set $\Lambda$ is called a repeller for $X$ if $\Lambda$ is an attractor for $X^{-1}$.

The set $\Lambda^{*}=M \backslash W^{s}(\Lambda)$ is a repeller $[2,4]$.

Proposition $2[2,4]$. An invariant set $\Lambda$ is an attractor if and only if there exists a neighborhood $U$ of $\Lambda$ such that

$$
X(c l U) \subset U, \Lambda=\bigcap_{n>0} X^{n}(U), W^{s}(\Lambda)=\bigcup_{n<0} X^{n}(U) .
$$

The set $U$ is called a fundamental neighborhood of $\Lambda$. We can say that each trajectory through $W^{s}(\Lambda) \backslash \Lambda$ begins in the repeller $\Lambda^{*}$ and finishes in the attractor $\Lambda[2,4]$. One of our aims is to construct the attractor, repeller and domain of attraction without any preliminary information about the dynamical system.

Definition 3. A sequence $\left\{x_{k}\right\}$, infinite in both directions, is called an $\varepsilon$-trajectory of $X$ if for any $k$ the distance between the image $X\left(x_{k}\right)$ and $x_{k+1}$ is less than $\varepsilon$ :

$$
\rho\left(X\left(x_{k}\right), x_{k+1}\right)<\varepsilon .
$$

If an $\varepsilon$-trajectory $\left\{x_{k}\right\}$ is periodic, that is, $x_{k+p}=x_{k}$ for some $p>0$, then it is called a $p$-periodic $\varepsilon$-trajectory and the points $x_{k}$ are called $(p, \varepsilon)$-periodic. We call a point $\varepsilon$-periodic if it is $p$-periodic for some period $p$.

In the majority of cases, exact trajectories of the system are not known, and in fact we find only $\varepsilon$-trajectories for sufficiently small, positive $\varepsilon$. As may be expected, the properties of the attractor and its domain of attraction persist for the $\varepsilon$-trajectories.

Proposition 3. Let $\Lambda$ be an attractor, $x \in W^{s}(\Lambda)$ and a neighborhood $V$ of $\Lambda$ be given. Then

1) there exist a neighborhood $U^{*}$ of $\Lambda, U^{*} \subset V$ and $\varepsilon_{1}>0$ such that each positive $\varepsilon_{1}$-semi-trajectory through $U^{*}$ remains in $U^{*}$,

2) there exists $\varepsilon_{2}$ such that each $\varepsilon_{2}$-trajectory through $V\left(\varepsilon_{2}, x\right)$ reaches $U^{*}$.

Proof. Let $U$ be a fundamental neighborhood of $\Lambda$ and $V$ be a neighborhood of $\Lambda$. According to Proposition 2, for $V$ there exists $k>0$ such that $X^{k}(U) \subset V$ and $c l X^{k+1}(U) \subset X^{k}(U)$. Set $U^{*}=X^{k}(U)$. The distance

$$
\rho\left(\operatorname{cl} X\left(U^{*}\right), M \backslash U^{*}\right)=\min \left(\rho(x, y), x \in \operatorname{cl} X\left(U^{*}\right), y \in M \backslash U^{*}\right)
$$

is positive, because $U^{*}$ is an open set and $c l X\left(U^{*}\right) \subset U^{*}$. Set

$$
\varepsilon_{1}=\rho\left(\operatorname{cl} X\left(U^{*}\right), M \backslash U^{*}\right) .
$$

In this case, the $\varepsilon_{1}$-neighborhood of $X\left(U^{*}\right)$ is contained in $U^{*}$. We must show that any positive $\varepsilon_{1}$-semi-trajectory through $U^{*}$ remains in $U^{*}$. In fact, let $x_{k}, x_{k+1}$ be a pair of consecutive points of an $\varepsilon_{1}$-semi-trajectory and let $x_{k} \in U^{*}$. Since $\rho\left(X\left(x_{k}\right), x_{k+1}\right)<\varepsilon_{1}$, the point $x_{k+1}$ is in the $\varepsilon_{1}$-neighborhood of $X\left(U^{*}\right)$. It follows that $x_{k+1} \in U^{*}$.

Let $x$ be a point in $W^{s}(\Lambda)$. We prove by contradiction that there is an $\varepsilon_{2}>0$ such that each $\varepsilon_{2}$-trajectory through $V\left(\varepsilon_{2}, x\right)$ reaches $U^{*}$. Suppose that for each $\varepsilon>0$ there exists a positive $\varepsilon$-semi-trajectory $w(\varepsilon)$ through $V(\varepsilon, x)$ which misses $U^{*}$. Let $\varepsilon_{n} \rightarrow 0$ and $\left\{w\left(\varepsilon_{n}\right)\right\}$ be the sequence of the positive $\varepsilon_{n}$-semi-trajectories described above. Since the sequence $\left\{w\left(\varepsilon_{n}\right)\right\}$ is in the compact $M$, there is a converging subsequence $w\left(\varepsilon_{n_{k}}\right) \rightarrow w$. 
The trajectory $w$ passes through $x$ and is outside $U^{*}$. We come to a contradiction, because the trajectory $w$ has to finish in $\Lambda \subset U^{*}$.

Corollary 1. It is evident that $\varepsilon=\min \left(\varepsilon_{1}, \varepsilon_{2}\right)$ satisfies both of the conclusions of the proposition, i.e., each $\varepsilon$-trajectory through $V(\varepsilon, x)$ reaches $U^{*}$ and remains there.

Proposition 4. Let $V_{1}$ be an arbitrarily small neighborhood of an attractor $\Lambda$ and let $V_{2}$ be an arbitrarily large neighborhood such that

$$
\Lambda \subset V_{1} \subset V_{2} \subset \operatorname{cl} V_{2} \subset W^{s}(\Lambda) .
$$

Then there exist $\varepsilon>0$ and neighborhoods $U_{1}, U_{2}$ of $\Lambda$,

$$
\Lambda \subset U_{1} \subset V_{1} \subset V_{2} \subset U_{2} \subset c l U_{2} \subset W^{s}(\Lambda)
$$

such that

1) each $\varepsilon$-trajectory through $U_{2} \backslash U_{1}$ starts outside $U_{2}$ and finishes in $U_{1}$,

2) each positive $\varepsilon$-semi-trajectory through $U_{1}$ remains there,

3) each negative $\varepsilon$-semi-trajectory through $M \backslash$ clU $U_{2}$ remains there.

Proof. By Proposition 3, for the neighborhood $V_{1}$ there exist $\varepsilon_{1}$ and a neighborhood $U_{1}$ such that $U_{1} \subset V_{1}$ and each positive $\varepsilon_{1}$-semi-trajectory through $U_{1}$ remains in $U_{1}$. Moreover, for a point $x \in W^{s}(\Lambda)$, there is $\varepsilon_{11}>0$ such that each $\varepsilon_{11}$-trajectory through $V\left(\varepsilon_{11}, x\right)$ finishes in $U_{1}$.

Recall that the set $\Lambda^{*}=M \backslash W^{s}(\Lambda)$ is a repeller for $X$ and an attractor for $X^{-1}$. The set $M \backslash c l V_{2}$ is a neighborhood of $\Lambda^{*}$ because $c l V_{2} \subset W^{s}(\Lambda)$. In view of the symmetry between attractor and repeller, there are $\varepsilon_{2}$ and a neighborhood $U^{*} \subset\left(M \backslash c l V_{2}\right)$ of $\Lambda^{*}$ such that each negative $\varepsilon_{2}$-semi-trajectory through $U^{*}$ remains in $U^{*}$. Set $U_{2}=M \backslash c l U^{*}$. Thus each negative $\varepsilon_{2}$-semi-trajectory through $U^{*}=M \backslash c l U_{2}$ remains there. Moreover, for any point $x \in W^{s}(\Lambda)$, there is $\varepsilon_{22}>0$ such that each $\varepsilon_{22}$-trajectory through $V\left(\varepsilon_{22}, x\right)$ starts outside $U_{2}$.

Let us consider the compact $K=\operatorname{cl}\left(U_{2} \backslash U_{1}\right)$. For any point $x \in K$ there are $\varepsilon_{11}$ and $\varepsilon_{22}$ described above. Set $\varepsilon(x)=\min \left\{\varepsilon_{11}, \varepsilon_{22}\right\}$. According to the construction, each $\varepsilon(x)$ trajectory through $V(\varepsilon(x), x)$ starts outside $U_{2}$ and finishes in $U_{1}$. The family of the neighborhoods $\{V(\varepsilon(x), x) ; x \in K\}$ forms an open covering of the compact $K$. There exists a finite covering of $K$ by the neighborhoods $\left\{V\left(\varepsilon_{m}, x_{m}\right): \varepsilon_{m}=\varepsilon\left(x_{m}\right), m=3,4, \ldots, r\right\}$. We set $\varepsilon=\min \left(\varepsilon_{m}, m=1,2,3, \ldots, r\right)$. Since $\varepsilon \leq \varepsilon_{1}$, each positive $\varepsilon$-semi-trajectory through $U_{1}$ remains there. Since $\varepsilon \leq \varepsilon_{2}$, each negative $\varepsilon$-semi-trajectory through $M \backslash c l U_{2}=U^{*}$ remains there. Since $\varepsilon \leq \varepsilon_{m}, m=3, \ldots, r$, each $\varepsilon$-trajectory through $U_{2} \backslash U_{1}$ starts outside $U_{2}$ and finishes in $U_{1}$.

Denote the set of all $\varepsilon$-periodic points by $Q(\varepsilon)$. The set $Q(\varepsilon)$ is open. It is clear that if $\varepsilon_{1}$ is greater than $\varepsilon_{2}$ then every $\varepsilon_{2}$-trajectory is an $\varepsilon_{1}$-trajectory, hence

$$
Q\left(\varepsilon_{2}\right) \subset Q\left(\varepsilon_{1}\right), \quad \varepsilon_{2}<\varepsilon_{1} .
$$

Definition 4. A point $x$ is called chain recurrent if $x$ is $\varepsilon$-periodic for every positive $\varepsilon$. The set of chain recurrent points is called the chain recurrent set.

Let us denote the chain recurrent set by $Q$. It is not difficult to show that the chain recurrent set is invariant, closed and contains the returning trajectories of all types such as 

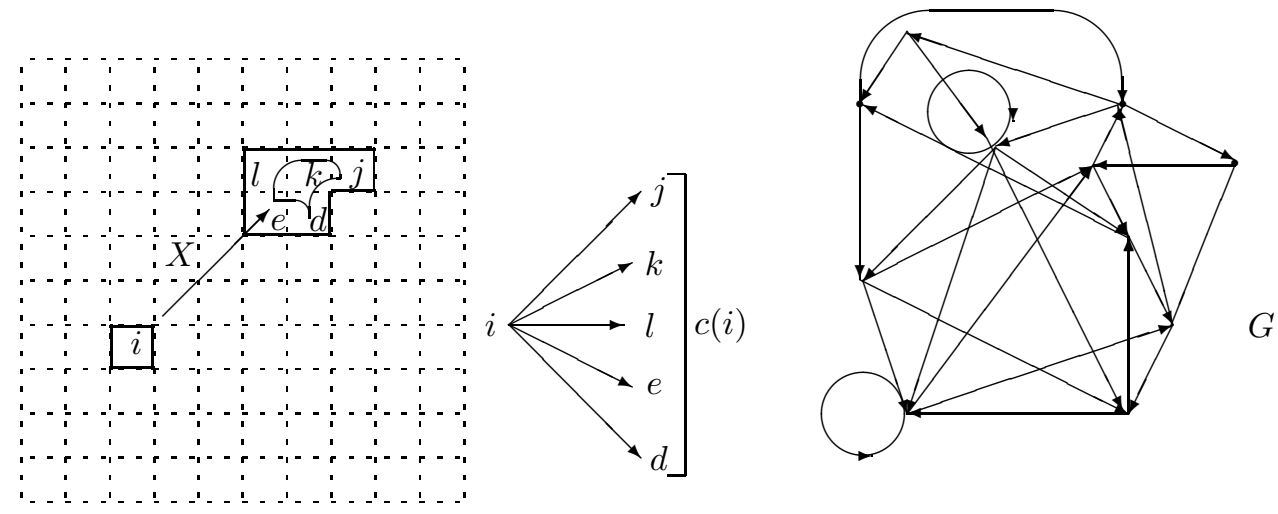

Fig. 1. Construction of the symbolic image

periodic, almost periodic, recurrent, homoclinic and other. It should be remarked that if a chain recurrent point is not periodic then there exists an arbitrarily small perturbation of the mapping $X$ in $C^{0}$-topology for which this point is periodic. One may say that a chain recurrent point becomes periodic under small $C^{0}$-perturbation. From the definition of the chain recurrent set it follows that

$$
Q=\lim _{\varepsilon \rightarrow 0} Q(\varepsilon)=\bigcap_{\varepsilon>0} Q(\varepsilon) .
$$

In other words the family $\{Q(\varepsilon), \varepsilon>0\}$ forms a base of neighborhoods of the chain recurrent set.

The construction of the symbolic image. Let $C=\{M(1), \cdots, M(n)\}$ be a finite covering of the domain $M$ by closed sets. The set $M(i)$ is called a cell of the covering. For any $i$ we define a covering $C(i)$ of the image $X(M(i))$, consisting of cells $M(j)$ whose intersections with $X(M(i))$ are non-empty:

$$
C(i)=\{M(j): M(j) \cap X(M(i)) \neq \emptyset\} .
$$

The cells of the covering $C(i)$ are called the image cells of $M(i)$, and we set

$$
c(i)=\{j: M(j) \cap X(M(i)) \neq \emptyset\} .
$$

Definition 5. Let $G$ be an oriented graph having $n$ vertices where each vertex $i$ corresponds to the cell $M(i)$. The vertices $i$ and $j$ are connected by an oriented edge $i \rightarrow j$ only in case if $j \in c(i)$. The graph $G$ is called the symbolic image of the mapping $X$ with respect to the covering $C$.

The oriented graph $G$ is uniquely determined by its transition matrix $\Pi=\left(\pi_{i j}\right)$, which has size $n \times n$. The element $\pi_{i j}=1$ if and only if $G$ contains the oriented edge $i \rightarrow j$, otherwise $\pi_{i j}=0$. A set of vertices $L$ is called a connected component if and only if each pair of vertices from $L$ is connected by a non-oriented path. In general, a graph can have several connected components. Symbolic dynamics involves the study of flows on such oriented graphs. Valuable information about a dynamical system may come from investigation of its symbolic image. It is easily seen that the symbolic image 
depends on the covering $C$. It is natural to consider the symbolic image as a finite, discrete approximation of the mapping $X$. This approximation is more precise if the mesh of the covering is smaller. Let

$$
\operatorname{diam} M(i)=\max (\rho(x, y): x, y \in M(i))
$$

be the diameter of the cell $M(i)$. Let $d$ be the largest diameter of the cells $M(i)$ of the covering $C$. Denote by $R_{i}$ the union of the cells $M(j)$ belonging to the covering $C(i)$ :

$$
R_{i}=\{\bigcup M(j): M(j) \in C(i)\}
$$

According to the definitions of the covering $C(i)$ and the largest diameter $d$, the set $R_{i}$ contains the image $X(M(i))$ and is contained in the closed $d$-neighborhood of the image:

$$
X(M(i)) \subset R_{i} \subset \operatorname{cl}\{d \text {-neighborhood of } X(M(i))\} .
$$

Denote by $q$ the largest diameter of the images $X(M(i)), i=1, \ldots, n$. We define the number $r$ as follows. If a cell $M(k)$ does not belong to the covering $C(i)$ then the distance

$$
\left.r_{i k}=\rho(X(M(i)), M(k))=\min (\rho(x, y): x \in X(M(i)), y \notin M(k))\right)
$$

is positive. Let $r$ be the minimum of such $r_{i k}$. Since the number of pairs $(i, k)$ described above is finite, $r$ is positive. Thus the number $r$ is the smallest distance between the images $X(M(i))$ and the cells $M(k)$ which do not intersect. The number $r$ is called the lower bound of the symbolic image $G$. It is clear that the lower bound depends on the covering $C$. By changing the covering $C$, we can construct a covering for which the lower bound $r$ is arbitrarily small. The next proposition describes some properties of the lower bound.

Proposition 5 [13]. If a point $x \in M(j)$ and $\rho(x, X(M(i)))<r$ then the cell $M(j)$ belongs to the covering $C(i)$.

The lower bound $r$ satisfies the inequality $r \leq d$.

The next corollary follows from the above proposition.

COROLlaRY 2. The set $R_{i}=\{\bigcup M(j): j \in c(i)\}$ contains the r-neighborhood of the image $X(M(i))$ :

$$
\{x: \rho(x, X(M(i)))<r\} \subset R_{i} .
$$

Corollary 3. The image $X(M(i))$ is contained in the interior, Int $R_{i}$, of $R_{i}$.

\section{Relation between the symbolic image and the dynamical system}

Definition 6. A sequence $\left\{z_{k}\right\}$, infinite in both directions, of vertices of the graph $G$ is called an admissible path or simply a path if for each $k$ the graph $G$ contains the edge $z_{k} \rightarrow z_{k+1}$.

We will call the path periodic if the sequence $\left\{z_{k}\right\}$ is periodic. There is a natural connection between the admissible paths on the symbolic image $G$ and the $\varepsilon$-trajectories of the homeomorphism $X$. It can be said that an admissible path is a trace of an $\varepsilon$ trajectory and the converse holds as well. However, there are some relationships between 
the parameters $d, q, r$ of the symbolic image and the number $\varepsilon$ for which these connections take place.

Proposition 6 [13].

1. If a sequence $\left\{z_{k}\right\}$ is a path on the symbolic image $G$ and $x_{k} \in M\left(z_{k}\right)$, then the sequence $\left\{x_{k}\right\}$ is an $\varepsilon$-trajectory of the homeomorphism $X$ for any $\varepsilon>q+d$. In particular, if the sequence $\left\{z_{k}\right\}$ is a periodic path on the symbolic image, then the sequence $\left\{x_{k}\right\}$ is an $\varepsilon$-periodic trajectory.

2. If a sequence $\left\{z_{k}\right\}$ is a path on the symbolic image $G$, then there exists a sequence $\left\{x_{k}\right\}, x_{k} \in M\left(z_{k}\right)$, which is an $\varepsilon$-trajectory of the homeomorphism $X$ for each $\varepsilon>d$.

3. If a sequence $\left\{x_{k}\right\}$ is an $\varepsilon$-trajectory of the homeomorphism $X, \varepsilon<r$ and $x_{k} \in$ $M\left(z_{k}\right)$, then the sequence $\left\{z_{k}\right\}$ is an admissible path on the symbolic image $G$. In particular, if the sequence $\left\{x_{k}\right\}$ is an $\varepsilon$-periodic trajectory, then the sequence $\left\{z_{k}\right\}$ is a periodic path on the symbolic image $G$.

Definition 7. A vertex of the symbolic image is called recurrent if a periodic path passes through it.

A pair of recurrent vertices $i, j$ are called equivalent if there is a periodic path through $i$ and $j$.

The recurrent vertices are uniquely defined by the non-zero diagonal elements of the powers of the transition matrix $\Pi^{m}, m \leq n$, where $n$ is the number of the covering cells [1]. According to the definition, the set of recurrent vertices decomposes into several classes $\left\{H_{k}\right\}$ of equivalent recurrent vertices. Let us consider a path $\omega$. Each nonrecurrent vertex $j$ may appear once in $\omega$. In fact, if the vertex $j$ appears two times then there is a path of the form $\omega_{1}=\{j, \ldots, j\} \subset \omega$. The path $\omega_{1}$ is periodic, and $j$ is recurrent. We come to a contradiction. It follows that each path $\omega=\left\{\ldots, i, j, \ldots, j^{*}, k, \ldots\right\}$ has a finite part, $\omega_{1}=\left\{j, \ldots, j^{*}\right\}$, which contains all nonrecurrent vertices from $\omega$. Thus $\omega$ begins in a class, $H_{l}$, of equivalent recurrent vertices and ends in a class, $H_{s}$, of equivalent recurrent vertices. It is not difficult to prove that if $\omega_{1} \neq \emptyset$ then $H_{l} \neq H_{s}$.

Denote by $P(d)$ the union of cells $M(i)$ for which the vertices $i$ are recurrent:

$$
P(d)=\{\bigcup M(i): i \text { are recurrent }\},
$$

where $d$ is the largest diameter of the cells $M(i)$. It should be noted that in fact the set $P$ depends on the covering $C$. However, in what follows we need only consider the dependence of $P$ on the largest diameter $d$. Let us denote by $T(d)$ the union of the cells $M(k)$ for which the vertices $k$ are not recurrent:

$$
T(d)=\{\bigcup M(k): k \text { are not recurrent }\} .
$$

THEOREM 1 [13].

1. The set $P(d)$ is a closed neighborhood of the chain recurrent set. Moreover, $P(d)$ consists of $\varepsilon$-periodic points for any $\varepsilon>q+d$, i.e.,

$$
P(d) \subset Q(\varepsilon), \varepsilon>q+d .
$$


2. The chain recurrent set $Q$ coincides with the intersection of the sets $P(d)$ for all positive d:

$$
Q=\bigcap_{d>0} P(d)
$$

3. The points of $T(d)$ are not chain recurrent. Moreover, if $\varepsilon<r$ there is no $\varepsilon$-periodic trajectory passing through points of $T(d)$, i.e.,

$$
Q(\varepsilon) \cap T(d)=\emptyset, \varepsilon<r .
$$

The set $T(d)$ is closed by construction and the pair $\{P(d), T(d)\}$ forms a closed covering of $M$. Hence the set $P(d) \backslash T(d)$ is an open neighborhood of the chain recurrent set $Q$. Theorem 1 leads us to the following inclusions

$$
Q \subset Q\left(\varepsilon_{1}\right) \subset M \backslash T(d)=P(d) \backslash T(d) \subset P(d) \subset Q\left(\varepsilon_{2}\right), \varepsilon_{1}<r<q+d<\varepsilon_{2} .
$$

Thus the set $P(d)$ is situated between the neighborhoods $Q\left(\varepsilon_{1}\right)$ and $Q\left(\varepsilon_{2}\right)$. However, the sets $P(d)$ for positive $d$ are not embedded in one another, in general. Theorem 1 makes it possible to localize the chain recurrent set with no preliminary information about the dynamical system [13].

Attractor, repeller and domain of attraction on the symbolic image. Let us consider a symbolic image $G$ with maximal diameter of the covering cells equal to $d$. Denote the set of vertices of $G$ by $\operatorname{Ver}(G)$. A set of vertices $L \subset \operatorname{Ver}(G)$ gives rise to a subgraph $G(L)$ which contains the vertices $L$ and the edges $i \rightarrow j$ if and only if the vertices $i$ and $j$ belong to $L$. We say that the set $L$ is invariant if for each vertex $i \in L$ there exist edges $j \rightarrow i$ and $i \rightarrow k$ in $G(L)$. In order to construct an invariant set we consider a path $\omega=\left\{\ldots, i_{-1}, i_{0}, i_{1}, \ldots\right\}$ infinite in both directions. The set of vertices $\operatorname{Ver}(\omega)$ of the path $\omega$ forms an invariant set because for each $i_{k} \in \omega$ there are edges $i_{k-1} \rightarrow i_{k}$ and $i_{k} \rightarrow i_{k+1}$. The same way, a family of paths $S=\{\omega\}$ gives the set of vertices, $\operatorname{Ver}(S)$, which is invariant. We can say that a set $L$ is invariant if for each vertex $i \in L$ there is an admissible path through $i$ lying in $L$. According to Proposition 6 , each trajectory $\left\{x_{k}\right\}$ of the homeomorphism $X$ generates a path $\left\{z_{k}: x_{k} \in M\left(z_{k}\right)\right\}$ on the symbolic image $G$. It immediately follows that an invariant set $\Lambda \subset M$ generates an invariant set of vertices of the form

$$
L(\Lambda)=\{z: M(z) \cap \Lambda \neq \emptyset\} .
$$

In particular the set $\operatorname{Ver}(G)$ is invariant. Let $L$ be an invariant set of vertices on the symbolic image $G$. The set of vertices

$$
\operatorname{En}(L)=\{i: i \notin L, \text { there exists an edge } i \rightarrow j, j \in L\}
$$

is called the entrance of $L$. The set of vertices

$$
E x(L)=\{i: i \notin L, \text { there exists an edge } j \rightarrow i, j \in L\}
$$

is called the exit of $L$.

Definition 8. We say that an invariant set $L \subset \operatorname{Ver}(G)$ is an attractor if $\operatorname{Ex}(L)=\emptyset$.

We say that an invariant set $L \subset \operatorname{Ver}(G)$ is a repeller if $\operatorname{En}(L)=\emptyset$. 
Let $L \subset \operatorname{Ver}(G)$ be an attractor. A (minimal) domain of attraction is the set of vertices

$$
D(L)=\{j \text { : each path through } j \text { finishes in } L\},
$$

i.e., for each path $\left\{\ldots, j, \ldots, i_{k}, \ldots\right\}$ there exists a number $K$ such that the vertices $i_{k}$, $k>K$, belong to $L$.

Proposition 7. Let $L \subset \operatorname{Ver}(G)$ be an attractor. Then

1. the vertices from $D(L) \backslash L$ are nonrecurrent,

2. the set of vertices $L^{*}=\operatorname{Ver}(G) \backslash D(L)$ is a repeller.

Proof. 1. If $i \in D(L) \backslash L$ is recurrent then there is a periodic path $\omega=\{i=$ $\left.i_{0}, \ldots, i_{m}=i\right\}$. Because $i \notin L$ the path $\omega$ does not finish in $L$. This means that $i$ does not belong to $D(L)$. We come to a contradiction. It follows that the vertices from $D(L) \backslash L$ are nonrecurrent.

2. If $L$ is a union of connected components then $D(L)=L$, and $L^{*}$ is also a union of connected components. In this case $L^{*}$ is a repeller. Suppose that $L$ is not a union of connected components, in particular, $L \neq \operatorname{Ver}(G)$. First we prove that $L^{*} \neq \emptyset$. If $L=D(L)$ then $L^{*}=\operatorname{Ver}(G) \backslash L \neq \emptyset$. Let $D(L) \backslash L \neq \emptyset$. Because the number of vertices is finite, the beginning of each path through $j \in D(L) \backslash L$ has a periodic part, i.e., the path is of the form $\left\{\ldots, i_{k}, \ldots, i_{l}, \ldots, j, \ldots\right\}$ with $i_{k}=i_{l}$. Since the exit of $L$ is empty, no vertex of the closed path $\left\{i_{k}, \ldots, i_{l}=i_{k}\right\}$ can belong to $L$. Because the vertices from $D(L) \backslash L$ are nonrecurrent, $i_{k} \notin D(L)$ and $i_{k} \in L^{*}$, i.e., $L^{*} \neq \emptyset$.

Now we prove by contradiction that $L^{*}$ is invariant. Let $i \in L^{*}$, and suppose there is no edge $i \rightarrow j$ for any $j \in L^{*}$. Then each edge $i \rightarrow k$ finishes in $D(L)$. In this case each path through $i$ finishes in $L$, i.e., $i \in D(L)$. It follows that $i \notin L^{*}$. We get a contradiction. The same way we can prove for each $i \in L^{*}$ there is an edge $j \rightarrow i, j \in L^{*}$.

Next we establish that $\operatorname{Ex}\left(L^{*}\right) \neq \emptyset$. Because $\operatorname{En}(L) \neq \emptyset$ there is an edge $i \rightarrow j, j \in$ $L, i \notin L$. It follows that either $i \in L^{*}$ or $i \in D(L) \backslash L$. In the first case, $j \in E x\left(L^{*}\right) \neq \emptyset$. In the second case, since the set $D(L) \backslash L$ consists of nonrecurrent vertices, each path through $i$ starts in $L^{*}$ and finishes in $L$. It follows that there is an edge $k \rightarrow l, k \in L^{*}, l \in D(L)$, i.e., $l \in E x\left(L^{*}\right) \neq \emptyset$.

Finally, we establish that $\operatorname{En}\left(L^{*}\right)=\emptyset$. Let $j \in E n\left(L^{*}\right)$. This means there is an edge $j \rightarrow i, j \in D(L), i \in L^{*}$. Because each path through $j$ finishes in $L$, each path through $i$ finishes in $L$ as well, i.e., $i \in D(L)$ and $i \notin L^{*}$. We get a contradiction.

The following proposition describes the structure of attractor on the symbolic image.

Proposition 8. Each attractor L consists only of some classes of equivalent recurrent vertices and all paths between these classes.

Proof. Let $L$ be an attractor; $i, j \in L ; \omega=\left\{i, \ldots i_{k}, \ldots, j\right\}$ be a path between $i$ and $j$. Since the exit $\operatorname{Ex}(L)$ is empty, each vertex $i_{k}$ from $\omega$ belongs to $L$. In particular if $i$ is a recurrent vertex, the class of recurrent vertices equivalent to $i$ lies in $L$. In a similar manner it also follows that all paths between these classes lie in $L$.

We must show there are no other types of vertices in $L$. Let $i \in L$ be a nonrecurrent vertex and $\omega$ be a path through $i$. Since $\operatorname{Ex}(L)=\emptyset, \omega$ finishes in $L$. Suppose there is no 
path through $i$ between the recurrent vertices from $L$. In this case every path $\omega$ which passes through $i$ passes between $L$ and $L^{*}$ with no recurrent vertices of $L$ preceding $i$ on $\omega$. Because there are only a finite number of nonrecurrent vertices, we can find one, say $k$, which is either $i$ itself or precedes $i$ on some $\omega$ and for which each edge $j \rightarrow i$ satisfies $j \notin L$. But then $L$ is not invariant. We get a contradiction.

Relation between the attractors of a dynamical system and of its symbolic image. As one would expect, there is a natural relation between the attractors of a dynamical system and the attractors of its symbolic image.

THEOREM 2. If $L$ and $D(L)$ are an attractor and its domain of attraction on a symbolic image $G$, then there are an attractor $\Lambda$ of the homeomorphism $X$ and its domain of attraction $W^{s}(\Lambda)$ such that the set

$$
U=\operatorname{Int}\{\bigcup M(i), i \in L\}
$$

is a fundamental neighborhood of $\Lambda$ and

$$
\{\bigcup M(j), j \in D(L)\} \subset W^{s}(\Lambda) .
$$

Proof. First we establish that $X(c l U) \subset U$. Since each cell $M(i)$ is closed, $c l U \subset$ $\{\cup M(i), i \in L\}$. By the definition of attractor, $\operatorname{Ex}(L)=\emptyset$. Hence, if $i \in L$ and there is an edge $i \rightarrow j$ then $j \in L$, i.e., the set $c(i)=\{j: M(j) \cap X(M(i)) \neq \emptyset\}$ lies in $L$. According to Corollary 3 we have

$$
X(c l U) \subset X\left(\bigcup_{i \in L} M(i)\right)=\bigcup_{i \in L} X(M(i)) \subset \bigcup_{i \in L}\left(i n t\left(\bigcup_{j \in c(i)} M(j)\right)\right) \subset \operatorname{int}\left(\bigcup_{j \in L} M(j)\right)=U .
$$

By Proposition 2 the set $\Lambda=\bigcap_{k>0} X^{k}(U)$ is an attractor of $X$, for which $U$ is a fundamental neighborhood.

Let $x \in\{\bigcup M(i): i \in D\}$ where $D$ is the domain of attraction for $L$. Consider the positive semi-trajectory $T^{+}(x)=\left\{X^{k}(x): k \in \mathbf{Z}^{+}\right\}$. According to Proposition $6, T^{+}(x)$ generates an admissible path $\omega=\left\{i_{k}: X^{k}(x) \in M\left(i_{k}\right), k \in \mathbf{Z}^{+}\right\}$on the symbolic image $G$ with $i_{0} \in D$. Because $D$ is a domain of attraction, the path $\omega$ finishes in $L$. This implies the existence of an integer $K$ such that $X^{k}(x) \in U$ for all $k>K$. Since $U$ is a fundamental neighborhood of $\Lambda, X^{k}(x) \rightarrow \Lambda$ as $k \rightarrow \infty$, i.e., $x \in W^{s}(\Lambda)$.

The following theorem shows that an attractor of a dynamical system and its domain of attraction can be defined as precisely as one likes by employing a symbolic image with covering cells of small enough diameter.

THEOREM 3. Let $\Lambda \subset M$ be an attractor, $V_{1}$ be its arbitrarily small neighborhood, and $V_{2}$ be an arbitrarily large neighborhood such that

$$
\Lambda \subset V_{1} \subset V_{2} \subset \operatorname{cl} V_{2} \subset W^{s}(\Lambda) .
$$

Then there exists $d_{0}>0$ such that each symbolic image $G$, with maximal diameter of covering cells $d<d_{0}$, has an attractor $L$ and its domain of attraction $D(L)$ such that

$$
\Lambda \subset\{\bigcup M(i), i \in L\} \subset V_{1} \subset V_{2} \subset\{\bigcup M(j), j \in D(L)\} \subset W^{s}(\Lambda) .
$$


Proof. According to Proposition 4, there are $\varepsilon_{0}>0$ and neighborhoods $U_{1}, U_{2}$ of $\Lambda$,

$$
\Lambda \subset U_{1} \subset V_{1} \subset V_{2} \subset U_{2} \subset \operatorname{cl} U_{2} \subset W^{s}(\Lambda)
$$

such that 1) each $\varepsilon_{0}$-trajectory through $U_{2} \backslash U_{1}$ starts outside $U_{2}$ and finishes inside $U_{1}$, 2 ) each positive $\varepsilon_{0}$-semi-trajectory through $U_{1}$ remains there, 3 ) each negative $\varepsilon_{0}$-semitrajectory through $M \backslash c l U_{2}$ remains there. Recall that $q\left(d_{0}\right)$ is the maximal diameter of images of cells under the condition that the diameter of each covering cell is less than $d_{0}$. Choose $d_{0}$ such that $q\left(d_{0}\right)+d_{0}=\varepsilon_{0}$. Since $M$ is compact and $X$ is continuous, the mapping $X$ is uniformly continuous. Let $\alpha(d)$ be the modulus of continuity of the mappings $X$ and $X^{-1}$. Hence, $\alpha(d) \rightarrow 0$ as $d \rightarrow 0$. We set $q(d)=\alpha(d)$. Fix some positive $d<d_{0}$. Consider a symbolic image $G$ with maximal diameter of cells $d$. According to Proposition 6 , each path $\left\{z_{k}\right\}$ on the symbolic image $G$ generates an $\varepsilon$-trajectory $\left\{x_{k}: x_{k} \in M\left(z_{k}\right)\right\}$ such that $q(d)+d<\varepsilon<\varepsilon_{0}$. Set

$$
\begin{aligned}
L_{1}=\left\{i: M(i) \subset U_{1}\right\}, & L_{2}=\left\{j: M(j) \cap \operatorname{cl}\left(U_{2} \backslash U_{1}\right) \neq \emptyset\right\}, \\
L_{3}=\{k: M(k) & \left.\subset M \backslash U_{2}\right\} .
\end{aligned}
$$

The neighborhood $U_{1}$ is constructed according to Proposition 3, as an image of a fundamental neighborhood. Hence we have the inclusion $\operatorname{cl} X\left(U_{1}\right) \subset U_{1}$. Moreover, the number $\varepsilon_{0}$ is found in the proofs of Propositions 3 and 4 with $\rho\left(\operatorname{cl} X\left(U_{1}\right), M \backslash U_{1}\right) \geq \varepsilon_{0}$. Since $\Lambda \subset X\left(U_{1}\right)$, the set of vertices $L_{0}=\{i: M(i) \cap \Lambda \neq \emptyset\}$ is in $L_{1}$. Because $\Lambda$ is an invariant set and each trajectory of $X$ generates a path on $G$, the set $L_{0}$ is invariant on $G$. Let $L$ be the maximal invariant set of vertices in $L_{1}$. Obviously, $L_{0} \subseteq L$. We must show that $L$ is an attractor. Consider any path $\omega=\left\{z_{k}\right\}$ through $i \in L$ and $\omega \not \subset L$. This means the path $\omega$ passes through a vertex $j$ such that $M(j) \cap\left(M \backslash U_{1}\right) \neq \emptyset$. According to Proposition 6 , each sequence $\left\{x_{k}: x_{k} \in M\left(z_{k}\right), z_{k} \in \omega\right\}$ is an $\varepsilon$-trajectory where $q(d)+d<\varepsilon<\varepsilon_{0}$. The sequence $\left\{x_{k}\right\}$ can be chosen so that $x_{i} \in U_{1}, x_{j} \in M \backslash U_{1}$. It is no loss of generality to set $0=\min \left(k: x_{k} \in M\left(z_{k}\right) \subset U_{1}, z_{k} \in \omega\right)$. By Proposition 4, the positive $\varepsilon$-trajectory $\left\{x_{k}, k>0\right\}$ remains in $U_{1}$. Moreover, since $x_{-1} \in M \backslash U_{1}$, the negative $\varepsilon$-trajectory $\left\{x_{k}, k<0\right\}$ has to start outside $U_{2}$ and must pass through $U_{2} \backslash U_{1}$. This means the path $\omega$ starts off $L$, passes through $L_{2}$ and ends in $L$. In this case, $E x(L)=\emptyset$, i.e., $L$ is an attractor. The same way we can prove that each path through $L_{2}$ ends in $L$. Denote by $D$ the domain of attraction for $L$. The above argument leads us to the conclusion that the domain of attraction $D \supset\left(L_{1} \cup L_{2}\right)$ and $U_{2} \subset\{\bigcup M(j), j \in D\} \subset W^{s}(\Lambda)$.

Thus the construction of an attractor of a dynamical system and its domain of attraction is reduced by Theorem 3 to the same task on a symbolic image.

Transition matrix and attractors. Let us introduce a quasi-order relation between the vertices of the symbolic image. We set $i \prec j$ if and only if there exists an admissible path of the form

$$
i=i_{0}, i_{1}, i_{2}, \ldots, i_{m}=j .
$$

Hence, a vertex $i$ is recurrent iff $i \prec i$, and a pair of recurrent vertices $i, j$ are equivalent if and only if $i \prec j \prec i$. 
Proposition 9 [1]. The vertices of a symbolic image $G$ can be renumbered such that

- the equivalent recurrent vertices are numbered with consecutive integers,

- the new numbers $i, j$ of other vertices are chosen such that $i<j$ if $i \prec j \nprec i$.

In other words, the transition matrix is of the form

$$
\Pi=\left(\begin{array}{ccccc}
\left(\Pi_{1}\right) & \cdots & \cdots & \cdots & \cdots \\
& \ddots & & & \\
0 & & \left(\Pi_{k}\right) & \ldots & \ldots \\
& \ddots & & \ddots & \\
0 & & 0 & & \left(\Pi_{s}\right)
\end{array}\right)
$$

where the elements under the diagonal are zeros, each diagonal block $\Pi_{k}$ corresponds to either a class of equivalent recurrent vertices $H_{k}$ or a nonrecurrent vertex. In the last case $\Pi_{k}$ coincides with a single zero. The renumbering described in Proposition 9 is not uniquely defined. Indeed, if there are no admissible paths from $i$ to $j$ and from $j$ to $i$, i.e., $i \nprec j, j \nprec i$, then the order between $i$ and $j$ is not fixed by Proposition 9 . In this case the order between the vertices $i, j$ may be arbitrarily chosen. From Propositions 8 and 9 it follows that for any attractor $L=\{i\}$, its domain of attraction $D(L)=\{j\}$, and corresponding repeller $L^{*}=\{k\}$, there is a renumbering such that

$$
k<j<i, \text { where } j \in D(L) \backslash L \text {. }
$$

In fact, as $i \in L, j \in D(L) \backslash L$ and $k \in L^{*}$ there are the relations

and conceivably there are the relations

$$
j \nprec k, i \nprec j,
$$

$$
k \prec j, j \prec i .
$$

This leads us to the renumbering of the form: $k<j<i$. But according to Proposition 7 , the vertices from $D(L) \backslash L$ are nonrecurrent. Thus, the transition matrix takes the form

$$
\Pi=\left(\begin{array}{ccccc}
\left(\Pi_{1}\right) & \ldots & \ldots & \ldots & \ldots \\
& 0 & \ldots & \ldots & \ldots \\
0 & & \ddots & & \\
& \ddots & & 0 & \ldots \\
0 & & 0 & & \left(\Pi_{2}\right)
\end{array}\right),
$$

where the blocks $\Pi_{1}, \Pi_{2}$ correspond to the repeller $L^{*}$ and the attractor $L$, respectively. Thus we can find the attractors of a symbolic image by representations of the transition matrix in the form (2). One should bear in mind that the transition matrix of the form (1) is not uniquely defined.

Construction of attractor, its domain of attraction and repeller of a dynamical system. In order to construct an attractor, its domain of attraction and a repeller, we apply the process of subdivision described in the localization algorithm for the chain recurrent set [13]. First, we consider a subdivision of a covering. The subdivision is the main step of the construction. 
Let $C=\{M(i)\}$ be a covering of $M$ and $G$ be the symbolic image for $C$. Suppose a new covering $N C$ is a subdivision of $C$, i.e., each cell $M(i)$ is subdivided. Denote by $N G$ the symbolic image for $N C$. It is convenient to designate the cells of the new covering as $m(i, k)$. So the cells $m(i, k), k=1,2, \ldots$, form a subdivision of the cell $M(i)$ :

$$
\bigcup_{k} m(i, k)=M(i) \text {. }
$$

In this case the vertices of the new symbolic image are denoted as $(i, k)$. It is possible that some cells of the new subdivision are really not subdivided, i.e., $m(i)=M(i)$, and the vertex $i$ is in $G$ and in $N G$ too. The described subdivision generates a natural mapping $H$ from $N G$ onto $G$ which takes the vertices $(i, k)$ to the vertex $i$. Since from $X(m(i, k)) \cap m(j, l) \neq \emptyset$ it follows that $X(M(i)) \cap M(j) \neq \emptyset$, the oriented edge $(i, k) \rightarrow$ $(j, l)$ is mapped onto the oriented edge $i \rightarrow j$. Hence, the mapping $H$ takes the oriented graph $N G$ into the oriented graph $G$.

Consider a symbolic image $G$ having an attractor $L$ with domain of attraction $D(L)$ and corresponding repeller $L^{*}$. Suppose that the cells $M(i), i \in L$ are subdivided and the other cells remain as before. The new symbolic image has the same repeller $L^{*}$ for which a new attractor $N L$ exists. The repeller $L^{*}$ is an attractor for the inverse orientation. Under the inverse orientation, $L^{*}$ has a different domain of attraction on $G$ and $N G$. These domains are $D\left(L^{*}\right)=\operatorname{Ver}(G) \backslash L$ on $G$ and $N D\left(L^{*}\right)=\operatorname{Ver}(N G) \backslash N L$ on $N G$. Since only the cells $M(i), i \in L$ are subdivided, the restrictions of the symbolic images $G$ and $N G$ on $\operatorname{Ver}(G) \backslash L$ coincide. This leads us to the conclusion that $D\left(L^{*}\right) \subset N D\left(L^{*}\right)$ and the image $H(N L)$ is in $L$. Hence, the new attractor $N L$ is contained in the set of vertices $\{(i, k): i \in L\}$. So we get the inclusion

$$
\{\bigcup M(i), i \in L\} \supset\{\bigcup m(i, k),(i, k) \in N L\} .
$$

The domain of attraction for the new attractor $N L$ is $D(N L)=\operatorname{Ver}(N G) \backslash L^{*}$. Hence, we have

$$
\{\bigcup M(j), j \in D(L)\} \subset\{\bigcup m(j, l),(j, l) \in D(N L)\} .
$$

Now consider the second subdivision such that the cells $M(j), j \in L^{*}$ are subject to subdivision. The same way we obtain the inclusions

$$
\begin{gathered}
A_{1}=\{\bigcup M(i), i \in L\} \supset\{\bigcup m(i, k),(i, k) \in N L\}=A_{2}, \\
W_{1}=\{\bigcup M(j), j \in D(L)\} \subset\{\bigcup m(e, l),(e, l) \in D(N L)\}=W_{2}, \\
R_{1}=\left\{\bigcup M(k), k \in L^{*}\right\} \supset\left\{\bigcup m(k, q),(k, q) \in N L^{*}\right\}=R_{2},
\end{gathered}
$$

where $N L^{*}$ is the new repeller. We can consider each subdivision as three successive subdivisions: a) a subdivision of the cells $M(i), i \in L$; b) a subdivision of the cells $M(k), k \in L^{*}$, and c) a subdivision of the cells $M(j), j \in D(L) \backslash L$. In this case we come to the same inclusions. Note the equalities $A_{1}=A_{2}, R_{1}=R_{2}$ and $W_{1}=W_{2}$ hold under the subdivision of the cells $M(j), j \in D(L) \backslash L$. In fact, in this case the attractor $L$ and the repeller $L^{*}$ do not change. Hence, the new domain of attraction is $N D(L)=\{(i, k): i \in D(L)\}$ and $N D(L) \backslash L=\{(j, k): j \in D(L) \backslash L\}$. Since $\bigcup_{k} m(j, k)=M(j)$, we have $W_{1}=W_{2}$. 
Let us consider a covering $C$ and the corresponding symbolic image $G$. Suppose we pick an attractor $L$ on $G$. Choose a sequence of subdivisions such that the maximal diameter $d$ of covering cells tends to zero. We get a sequence of contracted sets $A_{1}, A_{2}, \ldots$, a sequence of contracted sets $R_{1}, R_{2}, \ldots$ and a sequence of extended sets $W_{1}, W_{2}, \ldots$ From Theorems 2 and 3 it follows that there exists an attractor $\Lambda$, its domain of attraction $D(\Lambda)$ and the corresponding repeller $\Lambda^{*}$ such that

$$
\lim _{s \rightarrow \infty} A_{s}=\Lambda, \lim _{s \rightarrow \infty} W_{s}=W^{u}(\Lambda), \lim _{s \rightarrow \infty} R_{s}=\Lambda^{*} .
$$

Moreover, from Theorem 3 it follows that each attractor of the homeomorphism $X$ can be constructed by the described process. Thus we come to the following

Algorithm for the construction of the attractor, its domain of attraction and the repeller of a dynamical system.

1) Let $C$ be a covering of $M$ by cells having a small enough maximal diameter $d$. The symbolic image $G$ is constructed for the given covering.

2) The attractor $L$, its domain of attraction $D(L)$ and repeller $L^{*}$ are recognized.

3) The sets

$$
\begin{gathered}
A=\{\bigcup M(i), i \in L\}, \\
W=\{\bigcup M(j), j \in D(L)\}, \\
R=\left\{\bigcup M(k), k \in L^{*}\right\}
\end{gathered}
$$

are defined. Let $d=\max \left\{\operatorname{diam} M(i), \operatorname{diam} M(k): i \in L, k \in L^{*}\right\}$.

4) The cells corresponding to the attractor $L$ and the repeller $L^{*}$ are subdivided, and a new covering is found.

5) The symbolic image is constructed for the new covering.

6) Return to the second step.

Repeating this process we obtain sequences of sets $A_{1}, A_{2}, \ldots ; W_{1}, W_{2}, \ldots ; R_{1}, R_{2}, \ldots$, and a sequence of numbers $d_{1}, d_{2}, \ldots$. Above we proved

THEOREM 4. 1. The described algorithm gives the sequences of embedded sets

$$
A_{1} \supset A_{2} \supset \cdots, \quad W_{1} \subset W_{2} \subset \cdots, \quad R_{1} \supset R_{2} \supset \cdots .
$$

2. If $d_{s} \rightarrow 0$ as $s$ becomes infinite then

$$
\begin{gathered}
\lim _{s \rightarrow \infty} A_{s}=\Lambda \text { is an attractor, } \\
\lim _{s \rightarrow \infty} W_{s}=W^{s}(\Lambda) \text { is its domain of attraction, } \\
\lim _{s \rightarrow \infty} R_{s}=\Lambda^{*} \text { is the repeller corresponding to } \Lambda .
\end{gathered}
$$

3. Each attractor $\Lambda$ can be constructed by this algorithm.

Consider a limitation of the proposed algorithm. If an attractor $\Lambda$ is fixed then the maximal diameter $d$ for an initial covering is defined by $\Lambda$ according to Theorem 3 . So the choice of an initial covering requires some previous information. In reality, the attractor $\Lambda$ is defined by choice of the attractor $L$ on the initial symbolic image $G$. Subsequent 
subdivisions localize the attractor $\Lambda$. Now we consider the concept of filtration which helps select the initial choice.

\section{Filtrations}

Definition 9 [10]. A filtration for the homeomorphism $X$ is a finite sequence $F=\left\{U_{0}, U_{1}, \ldots, U_{m}\right\}$ of open sets such that $\emptyset=U_{0} \subset U_{1} \subset \cdots \subset U_{m}=M$ and for each $k=0,1, \ldots, m, X\left(c l U_{k}\right) \subset U_{k}$.

The second condition is a property of a fundamental neighborhood of attractor. See Proposition 2. The next proposition describes the structure of attractors generated by a filtration. In addition, a persistence property of filtration is given.

Proposition 10 [4]. For a given filtration $F$

1) the maximal invariant subset in $U_{k}, k=0,1, \ldots, m$

is an attractor for $X$ and

$$
A_{k}=\left\{\bigcap X^{n}\left(U_{k}\right): n \in Z^{+}\right\}
$$

$$
\emptyset=A_{0} \subset A_{1} \subset \cdots \subset A_{m}=M,
$$

2) there is a neighborhood $V$ of $X$ in $C^{0}$ topology such that the sequence $F$ is a filtration for any map $Y \in V$.

The maximal invariant subset in $U_{k} \backslash U_{k-1}$ is denoted

$$
K_{k}(F)=\left\{\bigcap X^{n}\left(U_{k} \backslash U_{k-1}\right): n \in Z\right\},
$$

and we set $K(F)=\left\{\bigcup K_{k}(F): k=1, \cdots, m\right\}$.

We wish to establish that the chain recurrent set $Q$ lies in $K(L)$. Consider an attractor $\Lambda$, its domain of attraction $W^{s}(\Lambda)$ and the repeller $\Lambda^{*}$ corresponding to $\Lambda$. First we prove that each point $x$ from $W^{s}(\Lambda) \backslash \Lambda$ is nonrecurrent. In fact, there is a neighborhood $V$ such that $x \in W^{s}(\Lambda) \backslash c l V$. According to Proposition 3, for the neighborhood $V$ and the point $x \in W^{s}(\Lambda) \backslash \Lambda$, there is $\varepsilon>0$ such that each positive $\varepsilon$-trajectory through $x$ reaches $V$ and remains in $V$. Hence, there is no periodic $\varepsilon$-trajectory through $x$, and the point $x$ is not recurrent. This means that

$$
Q \cap\left(W^{s}(\Lambda) \backslash \Lambda\right)=\emptyset .
$$

Consider a filtration $F=\left\{U_{0}, U_{1}, \ldots, U_{m}\right\}$. Fix a chain recurrent point $x$. From the above it follows that if the point $x$ does not lie in the attractor $A_{k}=\bigcap_{n>0} X_{n}\left(U_{k}\right)$ then $x \notin W^{s}\left(A_{k}\right)$. Since the attractors $A_{0}, A_{1}, \ldots, A_{m}$ form a sequence of extended sets, there exists an attractor $A_{l}$ such that $x \in A_{l}$ and $x \notin A_{l-1}$. Because each $U_{k}$ is a fundamental neighborhood of $A_{k}$,

$$
A_{l}=\bigcap_{n} X^{n}\left(U_{l}\right), W^{s}\left(A_{l-1}\right)=\bigcup_{n} X^{n}\left(U_{l-1}\right) .
$$

Then $x \in \bigcap_{n} X^{n}\left(U_{l}\right)$ and $x \notin W^{s}\left(A_{l-1}\right)=\bigcup_{n} X^{n}\left(U_{l-1}\right)$. From the equality $(A \backslash B) \cap$ $(C \backslash D)=(A \cap C) \backslash B \cup D$ it follows that

$$
K_{l}(F)=\bigcap_{n} X^{n}\left(U_{l} \backslash U_{l-1}\right)=\bigcap_{n} X^{n}\left(U_{l}\right) \backslash \bigcup_{n} X^{n}\left(U_{l-1}\right)=A_{l} \backslash W^{s}\left(A_{l-1}\right) \ni x .
$$

Thus $Q \subset K(F)=\bigcup_{k} K_{k}(F)$. 
We call a filtration $F$ fine if $K(F)=Q$. (It should be noted that in [10] a filtration $F$ is called fine if $K(F)$ coincides with the nonwandering set.) If $X$ does not admit a fine filtration, it is common practice to consider a sequence $\left\{F_{l}: l=1,2, \ldots\right\}$ of filtrations for which the sequence $K\left(F_{l}\right)$ tends to $Q$ as $l \rightarrow \infty$.

Definition 10. A filtration $F^{*}=\left\{U_{0}^{*}, \cdots, U_{p}^{*}\right\}$ refines a filtration $F=\left\{U_{0}, \cdots, U_{q}\right\}$ if for each $\alpha=1, \cdots, p$ there exists $\beta(\alpha), 1 \leq \beta(\alpha) \leq q$ such that

$$
U_{\alpha}^{*} \backslash U_{\alpha-1}^{*} \subset U_{\beta(\alpha)} \backslash U_{\beta(\alpha)-1} .
$$

A sequence $F_{1}, F_{2}, \ldots$ of filtrations for $X$ is said to be fine if $F_{k+1}$ refines $F_{k}$ and

$$
\bigcap_{k} K\left(F_{k}\right)=Q
$$

A fine sequence of filtrations is seen to control the growth of $Q$ under perturbation of the dynamical system. More precisely, if $F_{1}, F_{2}, \ldots$ is a fine sequence of filtrations then according to Proposition 10 for a finite sequence of filtrations $F_{1}, F_{2}, \ldots, F_{l}$ there is a neighborhood $V$ of $X$ in $C^{0}$ topology such that the sequence $F_{1}, F_{2}, \ldots, F_{l}$ is a refined sequence of filtrations for each map $Y \in V$. In the next section we prove that for any homeomorphism $X$ there exists a fine sequence of filtrations. Moreover, the fine sequence of filtrations can be constructed by a special sequence of symbolic images generated by the subdivision process.

\section{Filtration on a symbolic image}

Definition 11. A finite sequence $\Phi=\left\{B_{0}, B_{1}, \ldots, B_{m}\right\}$ of vertex sets on a symbolic image $G$ is called a filtration if

$$
\emptyset=B_{0} \subset B_{1} \subset \cdots \subset B_{m}=\operatorname{Ver}(G)
$$

and for each $B_{k}, k=1,2, \ldots, m$, if the beginning vertex $i$ of an edge $i \rightarrow j$ lies in $B_{k}$ then the end vertex $j$ lies in $B_{k}$ as well.

The second condition means that there is no exit from $B_{k}$. Let $L_{k}$ be a maximal invariant set in $B_{k}$.

Proposition 11. Each maximal invariant set $L_{k} \subset B_{k}$ is an attractor and

$$
\emptyset=L_{0} \subset L_{1} \subset \cdots \subset L_{m}=\operatorname{Ver}(G) .
$$

Proof. Let us fix some $k=1,2, \ldots, m$ and establish by contradiction that the exit $\operatorname{Ex}\left(L_{k}\right)$ is empty. Let $i \rightarrow j$ be an edge of $G$ such that $i \in L_{k}$ and $j \notin L_{k}$. Consider a path $\omega_{1}=\{\ldots, i, j, p, \ldots\}$ through the edge $i \rightarrow j$. Since there is no exit from $B_{k}$, the positive semi-path $\omega^{+}=\{i, j, p, \ldots\}$ lies in $B_{k}$. Since $i \in L_{k}$ and $L_{k}$ is invariant, there is a path $\omega_{2}=\{\ldots, q, i, \ldots\}$ through $i$ which is in $L_{k}$. In particular, the negative semi-path $\omega^{-}=\{\ldots, q, i\}$ lies in $B_{k}$. Hence the path $\omega=\omega^{-} \omega^{+}=\{\ldots, q, i, j, p, \ldots\}$ lies in $B_{k}$. Because $L_{k}$ is a maximal invariant set in $B_{k}, \omega$ lies in $L_{k}$. In particular, $j \in L_{k}$. We get a contradiction. Thus $\operatorname{Ex}\left(L_{k}\right)=\emptyset$, and each $L_{k}$ is an attractor.

The inclusions (5) follow from the inclusions (4) and the definition of the attractors $L_{k}$. 
From the proof it follows that for each $k$, the set $B_{k}$ corresponds to a fundamental neighborhood of an attractor.

Let $\Phi=\left\{B_{0}, B_{1}, \ldots, B_{m}\right\}$ be a filtration on a symbolic image. A maximal invariant subset of $B_{k} \backslash B_{k-1}$ is denoted $J_{k}(\Phi)$ and we set $J(\Phi)=\bigcup_{k} J_{k}(\Phi)$. We wish to establish that the set of recurrent vertices $R V$ lies in $J(\Phi)$. Fix a recurrent vertex $i$ and denote by $H(i)$ the class of recurrent vertices equivalent to $i$. From the inclusions (4) it follows that there is a set $B_{l}$ such that $i \in B_{l}$ and $i \notin B_{l-1}$. The set $B_{l}$ is a subset of the domain of attraction $D\left(L_{l}\right)$, because $B_{l}$ has no exit and $L_{l}$ is a maximal invariant set in $B_{l}$. From Proposition 8 it follows that $H(i) \subset L_{l}$ and $H(i) \cap B_{l-1}=\emptyset$. Hence, $H(i) \subset B_{l} \backslash B_{l-1}$. The set $H(i)$ is invariant. Since $J_{l}(\Phi)$ is a maximal invariant set in $B_{l} \backslash B_{l-1}$, the class $H(i)$ lies in $J_{l}(\Phi)$. Thus we have $R V \subset J(\Phi)$.

We will call a filtration $\Phi$ fine if $R V=J(\Phi)$. Let us establish that there exists a fine filtration on any symbolic image. The classes of equivalent recurrent vertices are denoted $H_{p}, p=1, \ldots, s$. We set $H_{p} \prec H_{q}$ if and only if there exists an admissible path from $H_{p}$ to $H_{q}$. Let the vertices of the symbolic image be renumbered according to Proposition 9 . In this case the transition matrix has the form

$$
\Pi=\left(\begin{array}{ccccc}
\left(\Pi_{1}\right) & \cdots & \ldots & \ldots & \ldots \\
& \ddots & & & \\
0 & & \left(\Pi_{p}\right) & \ldots & \ldots \\
& \ddots & & \ddots & \\
0 & & 0 & & \left(\Pi_{s}\right)
\end{array}\right),
$$

where the elements under the diagonal are zeros, each diagonal block $\Pi_{p}$ corresponds to either a class of equivalent recurrent vertices $H_{p}$ or a nonrecurrent vertex. In the last case $\Pi_{p}$ coincides with zero. Introduce the numbers $n\left(H_{p}\right)=\min \left\{i: i \in H_{p}\right\}$, and construct the sets

$$
E_{p}=\left\{i: i \geq n\left(H_{p}\right)\right\}, p=1, \ldots, s, E_{s+1}=\emptyset .
$$

Set $B_{k}=E_{p}$, where $p=s+1-k, k=0,1, \ldots, s$. We have $B_{0}=\emptyset$ and $B_{s}=\operatorname{Ver}(G)$.

Proposition 12. The finite sequence $\Phi=\left\{\emptyset=B_{0}, B_{1}, \ldots, B_{s}=\operatorname{Ver}(G)\right\}$, defined as above, is a fine filtration on the symbolic image $G$.

Proof. Fix a number $p$ between 0 and $s$, define $k=s+1-p$ and set $N\left(H_{p}\right)=$ $\max \left\{i: i \in H_{p}\right\}$. Consider a decomposition of the set of vertices $\operatorname{Ver}(G)$

$$
B_{k}=\left\{i: i \geq n\left(H_{p}\right)\right\}, \quad B_{k}^{*}=\left\{i: i \leq N\left(H_{p-1}\right)\right\}, \quad W_{k}=\left\{i: N\left(H_{p-1}\right)<i<n\left(H_{p}\right)\right\} .
$$

According to the construction, the set $B_{k}$ contains the classes $H_{q}, q \geq p$; the set $B_{k}^{*}$ contains the classes $H_{l}, l<p$ and the set $W_{k}$ contains only the nonrecurrent vertices. In this case the transition matrix takes the form

$$
\Pi=\left(\begin{array}{ccccc}
\left(Y^{*}\right) & \ldots & \ldots & \ldots & \ldots \\
& 0 & & & \\
0 & & \ddots & \ldots & \ldots \\
& \ddots & & 0 & \\
0 & & 0 & & (Y)
\end{array}\right),
$$


where the block $Y$ corresponds to $B_{k}$, and the block $Y^{*}$ corresponds to $B_{k}^{*}$. So $B_{k}$ and $B_{k}^{*}$ are an attractor and a repeller, respectively. The set $B_{k} \backslash B_{k-1}$ coincides with $H_{p} \cup W_{k-1}$. The maximal invariant set in $B_{k} \backslash B_{k-1}$ is the class $H_{p}$, i.e., $J_{k}(\Phi)=H_{p}$. Thus $J(\Phi)=\left\{\bigcup H_{p}: p=1, \ldots, s\right\}$ and the sequence $\Phi=\left\{B_{0}, B_{1}, \ldots, B_{s}\right\}$ is a fine filtration.

Proposition 13. Let $\Phi=\left\{B_{0}, B_{1}, \ldots, B_{s}\right\}$ be a filtration on the symbolic image $G$. Then the finite sequence $F=\left\{U_{0}, U_{1}, \ldots, U_{s}\right\}$, where $U_{k}=\operatorname{int}\left\{\bigcup M(i): i \in B_{k}\right\}$, is a filtration for the mapping $X$.

Proof. According to Proposition 11, each maximal invariant set $L_{k}$ in $B_{k}$ is an attractor on the symbolic image $G$. From Theorem 2, it follows that the set $\operatorname{int}\{\bigcup M(i)$ : $\left.i \in L_{k}\right\}$ is a fundamental neighborhood of an attractor $\Lambda_{k}$. We now establish that the set $U_{k}=\operatorname{int}\left\{\bigcup M(i): i \in B_{k}\right\}$ has the property: $X\left(c l U_{k}\right) \subset U_{k}$. Let us fix some point $x \in\left\{\bigcup M(i): i \in B_{k}\right\}$. According to Proposition 6, the image $X(x)$ generates an oriented edge $i \rightarrow j, x \in M_{i}, X(x) \in M_{j}$ on the symbolic image $G, i \in B_{k}$. Because $B_{k}$ has no exit, the vertex $j$ is in $B_{k}$. This means $X(x) \in\left\{\bigcup M(i): i \in B_{k}\right\}=c l U_{k}$. In reality the image $X(x)$ has to be in $U_{k}$. Otherwise $X(x) \in M(j), j \notin B_{k}$. This implies the existence of an edge $i \rightarrow j, j \notin B_{k}$ and we obtain a contradiction. So $X\left(c l U_{k}\right) \subset U_{k}$. From the inclusion $B_{k} \subset B_{k+1}$, it follows $U_{k} \subset U_{k+1}$. Since $B_{0}=\emptyset$ and $B_{s}=\operatorname{Ver}(G), U_{0}=\emptyset, U_{s}=M$. Thus the sequence $F=\left\{U_{0}, U_{1}, \ldots, U_{s}\right\}$ is a filtration for the homeomorphism $X$.

An algorithm for construction of a fine sequence of filtrations.

1) Let $C$ be an arbitrary finite covering of $M$ by closed cells. The symbolic image $G$ is constructed for the given covering.

2) The classes $H_{p}$ of equivalent recurrent vertices are recognized. Denote

$$
d=\max \{\operatorname{diam} M(i): i \text { are recurrent }\} .
$$

3) A fine filtration $\Phi=\left\{B_{0}, B_{1}, \ldots, B_{s}\right\}$ on the symbolic image $G$ is obtained by setting $B_{k}=\left\{i: i \geq n\left(H_{p}\right), p=s+1-k\right\}$.

4) The filtration $F=\left\{U_{0}, U_{1}, \ldots, U_{s}\right\}$ for the dynamical system is defined by setting $U_{k}=\left\{\bigcup M(i): i \in B_{k}\right\}$.

5) The cells corresponding to the recurrent vertices $\{M(i): i$ are recurrent $\}$ are subdivided. The new covering is found.

6) The symbolic image $G$ is constructed for the new covering.

7) Return to the second step.

The algorithm described gives a sequence of symbolic images $G_{m}$, fine filtrations $\Phi_{m}$ on each $G_{m}$, a sequence of filtrations $F_{m}$ on $M$ and a sequence of numbers $d_{m}$. The following theorem justifies the proposed algorithm.

Theorem 5. If, in the described algorithm, $d_{m} \rightarrow 0$ as $m$ becomes infinite then the sequence of filtrations $\left\{F_{m}\right\}$ is fine.

Proof. Suppose the subdivision algorithm yields a sequence of filtrations $\Phi_{m}=$ $\left\{B_{0}, B_{1}, \ldots, B_{s}\right\}$ on the symbolic images $G_{m}$. According to Proposition 12 each filtration $\Phi_{m}$ is fine. Hence a maximal invariant set in $B_{k} \backslash B_{k-1}$ coincides with a class of equivalent recurrent vertices $H_{p}$. By Proposition 13 each filtration $\Phi_{m}$ generates a filtration $F_{m}=$ $\left\{U_{0}, U_{1}, \ldots, U_{s}\right\}$ on the manifold $M$, where $U_{k}=\left\{\bigcup M(i): i \in B_{k}\right\}$. Since the maximal 
invariant set in $B_{k} \backslash B_{k-1}$ is $H_{p}, k=s+1-p$, a maximal invariant set $K_{k}\left(F_{m}\right)$ in $U_{k} \backslash U_{k-1}$ lies in $\left\{\bigcup M(i): i \in H_{p}\right\}$. It follows that the chain recurrent set $Q$ lies in $P_{m}=\{M(i): i$ are recurrent $\}$. From [13] it follows that for each $m$ the inclusion $P_{m} \supset P_{m+1}$ holds, and if $d_{m} \rightarrow 0$ as $m \rightarrow \infty$ then

$$
\lim _{m \rightarrow \infty} P_{m}=\bigcap_{m>0} P_{m}=Q
$$

Thus the sequence of filtrations $\left\{F_{m}\right\}$ is fine.

COROLlary 4. For each homeomorphism $X$ there exists a fine sequence of filtrations.

EXAMPLE. Let us consider the Hénon mapping

$$
f:(x, y) \rightarrow\left(1-x^{2}+y, \lambda x\right), \lambda=0.5
$$

on the plane $R^{2}$. The dynamical system generated by the Hénon mapping is numerically studied in the domain $[-2,2] \times[-1,1]$. The initial covering consists of 128 cells, which are $0.25 \times 0.25$ squares. Figure 2 presents the first three embedded neighborhoods of the Hénon attractor, which are obtained according to the localization process. The computer program realizing the localization algorithm was devised at the Laboratory of Nonlinear Analysis and Mathematical Modelling of St. Petersburg State Technical University.

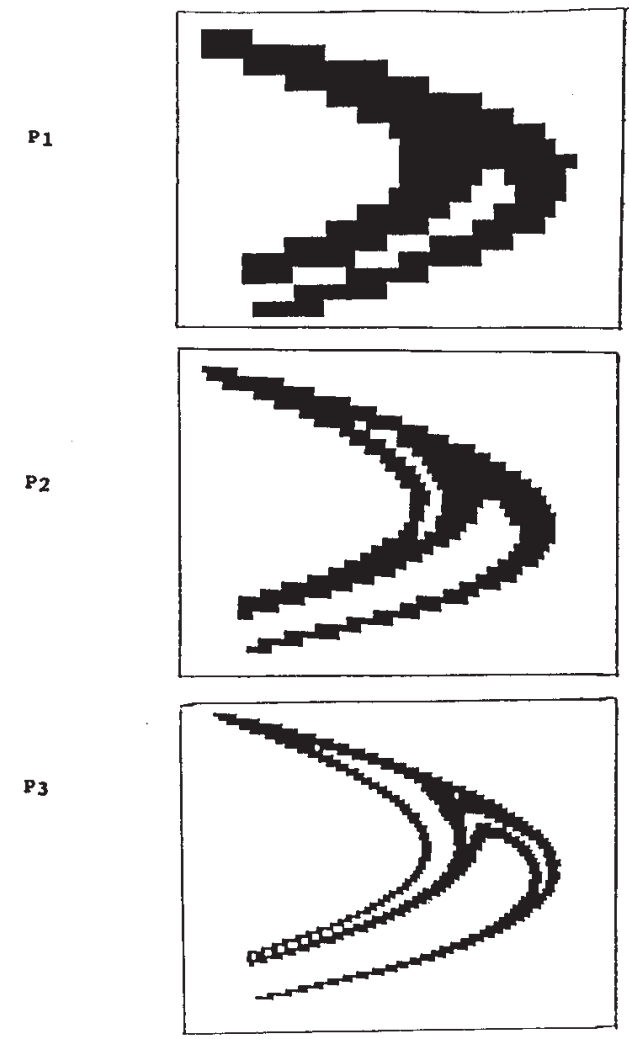

Fig. 2. The isolating neighborhoods for the Hénon attractor 


\section{References}

[1] V. M. Alekseev, Symbolic Dynamics, 11th Mathematical School, Kiev, 1976 (in Russian).

[2] N. P. Bhatia and G. P. Szegö, Stability theory of dynamical systems, N.Y., Springer, 1970.

[3] R. Bowen, Symbolic Dynamics, Amer. Math. Soc., Providence, R.I., vol. 8, 1982.

[4] I. U. Bronshtein, Nonautonomous dynamical systems, Kishinev, Shtinitsa, 1984 (in Russian).

[5] C. Conley, Isolated invariant sets and the Morse index, CBMS Regional Conference Series, 38, Amer. Math. Soc., Providence, 1978.

[6] P. Hartman, Ordinary Differential Equations, N.Y., 1964.

[7] C. S. Hsu, Cell-to-Cell Mappings, Springer-Verlag, N.Y., 1987.

[8] M. HuRley, Chain recurrence and attraction in non-compact spaces, Ergodic Theory and Dynamical Systems 11 (1991), 709-729.

[9] Z. Nitecki, Differentiable Dynamics, London, 1971.

[10] Z. Nitecki and M. Shub, Filtrations, decompositions, and explosions, Amer. J. Math. 97 (1975), 1029-1047.

[11] G. S. Osipenko, On a symbolic image of dynamical system, in: Boundary value problems, Perm, 1983, 101-105 (in Russian).

[12] G. S. OsipenKo, Verification of the transversality condition by the symbolic-dynamical methods, Differential Equations 26, 1126-1132; translated from Differentsial'nye Uravneniya 26 (1990), 1528-1536.

[13] G. S. Osipenko, The periodic points and symbolic dynamics, in: Seminar on Dynamical Systems, Birkhäuser Verlag, Basel, 1993, 261-267. 